\title{
Effects of polysaccharide rich extracts obtained from the brown seaweed Laminaria digitata on human microbiota in an in vitro model of the distal colon
}

Brown macroalgae are rich in a number of unique polysaccharides such as laminarin, fucoidan and alginate ${ }^{(1)}$. Several of these polysaccharides have demonstrated some activity with respect to stimulating beneficial gut bacteria and positively altering the metabolic activity of gut bacterial species ${ }^{(2,3,4)}$. Laminaria digitata (LD) is a common Irish brown seaweed and is a rich source of such polysaccharides. Therefore, the aim of this study was to extract these polysaccharides from LD and assess the effects on human faecal microbiota using an in vitro colonic model.

LD was harvested off the west coast of Ireland during July 2012. Polysaccharides were extracted using hot acid and ethanol precipitation. The extract was then either depolymerised (DE) or not (Crude, CE), before undergoing a simulated in vitro digestion followed by size exclusion chromatography to remove simple sugars. The extracts were fermented $(n=3)$ in anaerobic batch culture vessels with human faecal bacterial populations sourced from three healthy volunteers. Samples were removed at $0,5,10,24,36$ and 48 hour time points before undergoing culture dependent analysis of bifidobacteria and lactobacillus populations, and analysis of Short chain fatty acids using GC-FID.

Both extracts demonstrated some degree of fermentation, with both extracts significantly increasing total SCFA levels. Furthermore CE extract significantly increased both butyrate and propionate concentrations, while DE significantly increased propionate concentration. Neither extract was found to increase culturable Bifidobacteria and Lactobacillus populations. This study has demonstrated that both crude polysaccharides and depolymerised polysaccharides derived from LD affect the metabolic activities of human faecal microbiota. Further work is required to identify the microorganisms associated with these activities.
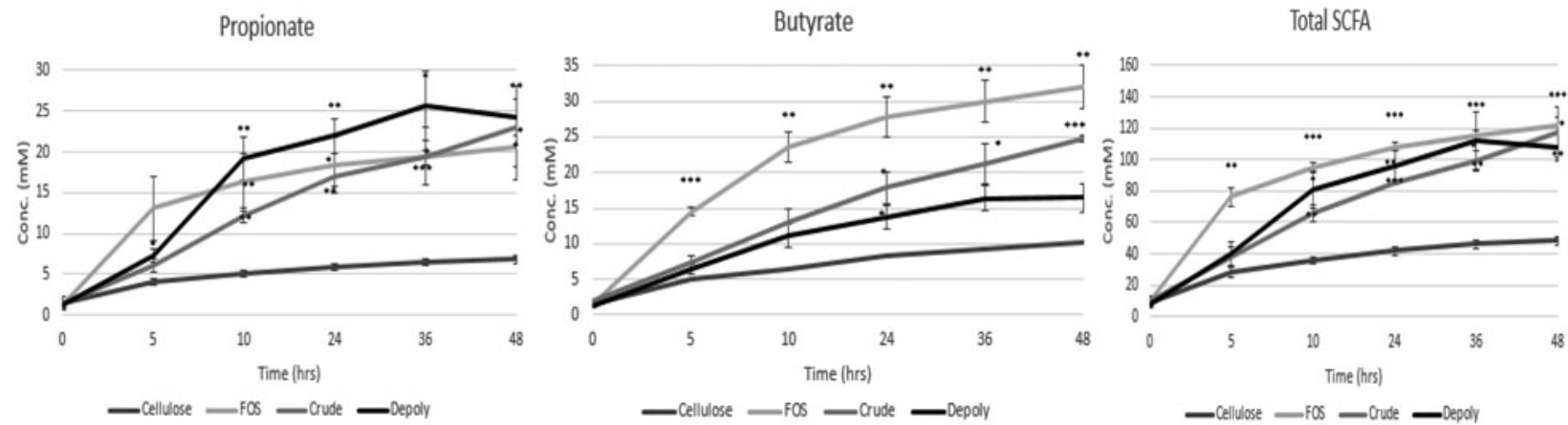

1. Gupta S \& Abu-Ghannam N (2011) Trends Food Sci Tec 22, 315-216.

2. An C, Kuda T, Yazaki T et al. (2013) Appl Environ Microbiol 79, 860-866.

3. Devillé C, Gharbi M, Dandrifosse G et al. (2007) J. Sci. Food Agric 87, 1717-1725.

4. O'Sullivan L, Murphy B, McLoughlin P et al. (2010) Mar Drugs 8, 2038-2064. 